Argonne

ANL-18/25

\title{
Acoustic Channel Link Models for Digital Communication Protocols
}

Transmission of Information by Acoustic Communication along Metal Pathways in Nuclear Facilities

Nuclear Science and Engineering Division 


\title{
About Argonne National Laboratory
}

Argonne is a U.S. Department of Energy laboratory managed by UChicago Argonne, LLC under contract DE-AC02-06CH11357. The Laboratory's main facility is outside Chicago, at 9700 South Cass Avenue, Argonne, Illinois 60439. For information about Argonne and its pioneering science and technology programs, see www.anl.gov.

\section{Document availability}

Online Access: U.S. Department of Energy (DOE) reports produced after 1991 and a growing number of pre-1991 documents are available free at OSTI.GOV

(http://www.osti.gov/), a service of the U.S. Dept. of Energy's Office of Scientific and

Technical Information

\section{Reports not in digital format may be purchased by the public from the} National Technical Information Service (NTIS):

U.S. Department of Commerce

National Technical Information Service

5301 Shawnee Rd

Alexandria, VA 22312

www.ntis.gov

Phone: (800) 553-NTIS (6847) or (703) 605-6000

Fax: (703) 605-6900

Email: orders@ntis.gov

\author{
Reports not in digital format are available to DOE and DOE contractors from the \\ Office of Scientific and Technical Information (OSTI): \\ U.S. Department of Energy \\ Office of Scientific and Technical Information \\ P.O. Box 62 \\ Oak Ridge, TN 37831-0062 \\ www.osti.gov \\ Phone: (865) 576-8401 \\ Fax: (865) 576-5728 \\ Email: reports@osti.gov
}

This report was prepared as an account of work sponsored by an agency of the United States Government. Neither the United States Government nor any agency thereof, nor UChicago Argonne, LLC, nor any of their employees or officers, makes any warranty, express or implied, or assumes any legal liability or responsibility for the accuracy, completeness, or usefulness of any information, apparatus, product, or process disclosed, or represents that its use would not infringe privately owned rights. Reference herein to any specific commercial product, process, or service by trade name, trademark, manufacturer, or otherwise, does not necessarily constitute or imply its endorsement, recommendation, or favoring by the United States Government or any agency thereof. The views and opinions of document authors expressed herein do not necessarily state or reflect those of the United States Government or any agency thereof, Argonne National Laboratory, or UChicago Argonne, LLC. 


\section{Acoustic Channel Link Models for Digital Communication Protocols}

Transmission of Information by Acoustic Communication along Metal Pathways in Nuclear Facilities

prepared by

Alexander Heifetz ${ }^{1}$, Jacey Young ${ }^{1,2}$, Xin Huang ${ }^{1,3}$, Sasan Bakhtiari ${ }^{1}$, Jafar Saniie ${ }^{3}$, and Richard B. Vilim ${ }^{1}$

${ }^{1}$ Nuclear Science Engineering Division, Argonne National Laboratory

${ }^{2}$ Department of Physics, St. Norbert College, De Pere, WI

${ }^{3}$ Department of Electrical and Computer Engineering, Illinois Institute of Technology, Chicago, IL

August 15, 2018 


\section{Table of Contents}

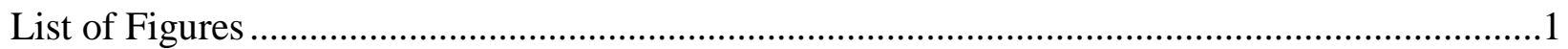

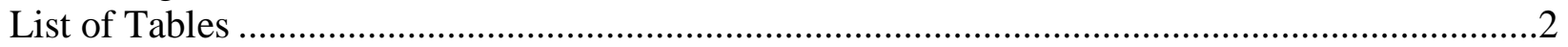

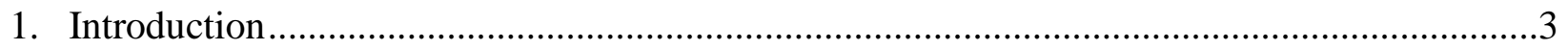

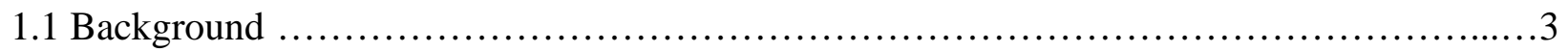

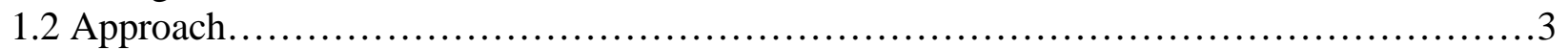

2. Computer Modeling of Acoustic Channel Link....................................................................5

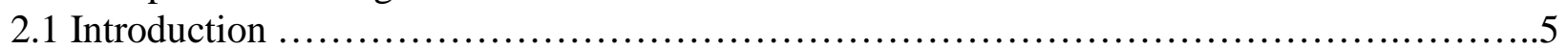

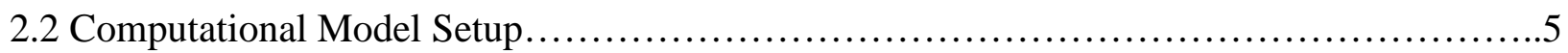

2.3 Two-Dimensional Radial Cross-Section Simulations ...........................................................6

2.4 Two-Dimensional Axial Cross-Section Simulations ..............................................................

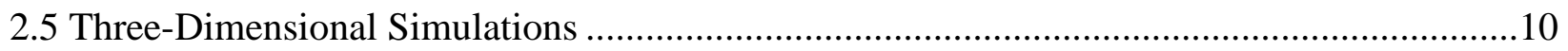

3. Resiliency of Communication System to Interferences............................................................11

3.1 Effect of Supporting Baffle Plates on Signal Transmission ...................................................11

3.2 Effect of Coupled Low Frequency Process Noise on Signal Transmission ............................12

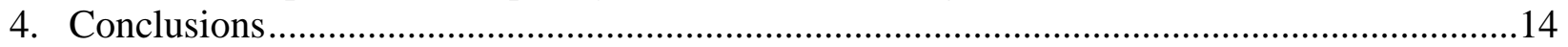

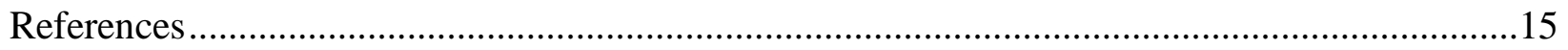




\section{List of Figures}

Figure 1 - Proposed acoustic communication system at a nuclear facility ................................ 4

Figure 2 - Pipe-transducer model setup for computer simulation........................................... 6

Figure 3 - Wave propagation along radial cross section of the pipe ........................................ 7

Figure 4 - Acoustic wave amplitude measured as displacement on pipe surface ........................ 8

Figure 5 - Acoustic wave intensity profile distribution in the axial direction............................ 9

Figure 6 - Multiple reflections of acoustic waves inside the wedge ....................................... 9

Figure 7 - Three dimensional simulation of refracted shear wave on the pipe .......................... 10

Figure 8 - Envisioned deployment at NPP and laboratory benchscale setup............................ 11

Figure 9 - Design and dimensions of each baffle plate ......................................................... 11

Figure 10 - Frequency-tunable mechanical shaker in contact with pipe .................................. 12

Figure 11 - Effect of low frequency vibrations on ultrasonic signal ................................... 13 


\section{List of Tables}

None... 


\section{Introduction}

\subsection{Background}

Integration of advanced communication technologies into nuclear facility operation has the potential for enhancing safety and accident-resiliency of the existing fleet of aging light water reactors, as well those of future advanced reactors. Conventional wired and wireless communication systems face implementation challenges at nuclear facilities due to the presence of thick reinforced concrete walls with steel liners throughout much of nuclear facility, especially the containment building. In addition, concerns related to security gaps in conventional communication networks have to be addressed when developing communication systems for nuclear facilities. To address communication technology gaps for nuclear energy, we are currently developing the acoustic communication (AC) hardware and network protocols for efficient and secure transfer of data, and will provide a preliminary experimental demonstration of the AC system prototype in a representative environment.

\subsection{Introduction}

In this project, we are developing a wireless communication system for a nuclear facility, in which information is carried with elastic waves propagating on metallic pipes. Traditionally, elastic wave propagation has been a frequent technique for non-destructive testing (NDT) of steel pipes. In the NDT methodology, elastic waves are launched into a steel pipe with an ultrasonic transducer. Material defects comparable in size to wavelength scatter ultrasonic waves, and analysis of scattered signal allows quantifying the defect and its location. As a variation on NDT technology, ultrasonic transducers placed at the ends of a pipe, and operating at wavelengths larger than typical microcrack sizes, can act as a transmitter and receiver of information. Such communication system would take advantage of the existing piping infrastructure to transmit information in and out of containment building, as shown in Figure 1. Furthermore, information sent over this system would only be accessible through direct physical contact with the pipes, thus establishing a protection layer against unauthorized eavesdroppers.

Because of limited bandwidth of ultrasonic transducers, on-off-keying OOK digital communication protocol was chosen for information transmission over an acoustic channel. Current work on the project is focused on investigating communication channel capacity (bit rate or duty cycle of the analog signal amplitude waveform), reliability (bit error rate) and energy efficiency (energy consumption per bit) of OOK. Design considerations of the OOK communication protocol are intimately connected to physical characteristics of the communication channel. This report addresses acoustic channel characterization through computer modelling of elastic wave coupling and propagation in the transducer/piping structure. In particular, computer models provide qualitative analysis of coupling losses and communication signal noise due to multiple scattering at the wedge/pipe interface. Also, 3-D simulations indicate that refracted shear wave a pipe generated with an angled wedge are torsional waves. In addition, proof-of-principle experimental studies were conducted to investigate performance of communication link under the 
conditions closely resembling a representative scenario. Anchoring baffle plates were added to the basic setup, and low frequency process noise was simulated by mechanically shaking the pipe.

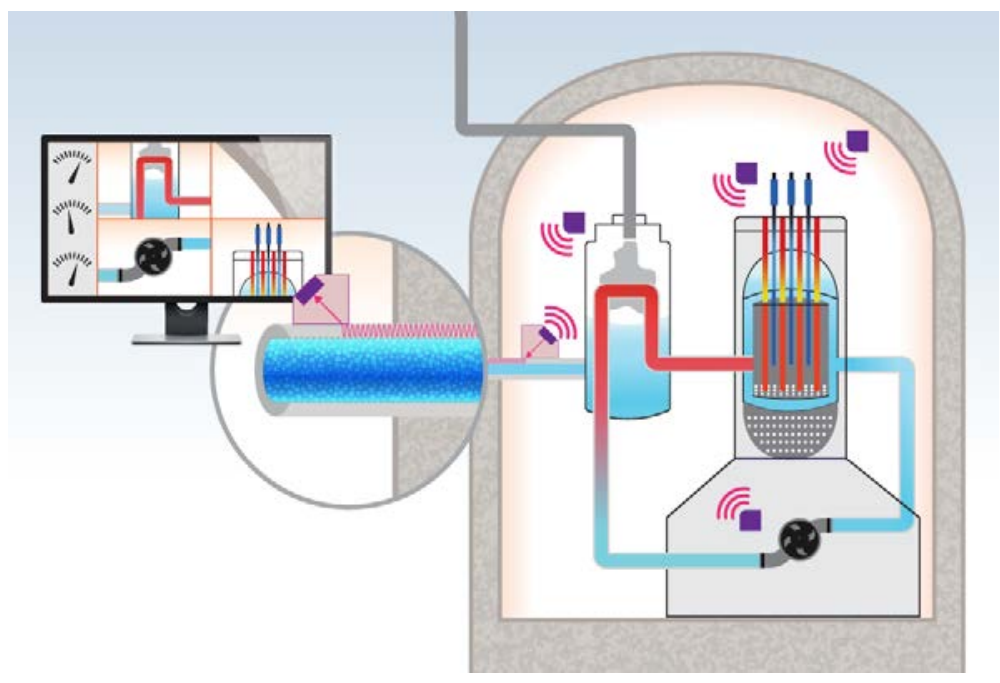

Figure 1 - Proposed acoustic communication system at a nuclear facility would transmit information on steel pipes already in place for nuclear reactor operation (Argonne 2018). 


\section{Computer Modeling of Acoustic Channel Link}

\subsection{Introduction}

Computer simulations were performed with COMSOL Multiphysics ${ }^{\mathrm{TM}}$ software package to provide support to ongoing experimental efforts described in previous reports [xx]. The objective of computer simulations described in this report is to develop better understanding of loss terms associated with coupling and propagation of elastic waves in the piping structure communication link. In prior work, we have modeled propagation of elastic waves generated on a pipe with a radially symmetric collar-type transducer (Bakhtiari 2017). However, we have subsequently determined that most likely conduits of elastic waves for information transmission out of the containment building are stainless steel pipes of the chemical volume and control system (CVCS) charging line (Heifetz 2017). These pipes are part of the thermal hydraulic system, and as such are enclosed by a layer of thermal insulation. Thermal insulation materials, such as mineral wool, are poor transmitters of acoustic waves. To achieve efficient coupling of acoustic waves into the pipe, the insulation material has to be removed for transducer to be directly in contact with the metal pipe. Thus, it is preferable to use a transducer with the smallest form factor so that minimal amount of thermal insulation needs to be removed and no thermal imbalances in the coolant system are created.

In this study, we have modeled propagation of refracted shear wave coupled into stainless steel pipe with an angled wedge. Such arrangement has a smaller form and requires removal of smaller amount of thermal insulation, as compared to ring of transducer. Two-dimensional simulations were developed to analyze propagation of elastic waves in the axial direction of the pipe, as well as in the radial cross section of the pipe. A three-dimensional simulation combined these results to visualize propagating both radially and axially around the pipe. The 3-D simulations indicate that the refracted shear waves on a pipe are created in a torsional mode.

\subsection{Computational Model Setup}

Software package COMSOL Multiphysics ${ }^{\mathrm{TM}}$ was used to model elastic waves propagating on a steel pipe structure for 2-D and 3-D computational model cases. Computer simulations of the general setup shown in Figure 2 were performed using the Solid Mechanics Module of COMSOL. The acoustic wave transducer was modeled as a PZT device. Although COMSOL library contains a module simulating PZT performance, we have determined through simulations that using the library module is computationally expensive. Instead, in this project we developed a computationally efficient model of a source of longitudinal acoustic waves. In this model, acoustic waves were generated with a rectangular iron block vibrating at an ultrasonic frequency against a plastic wedge. The amplitude of displacement of the iron block is on the order of a micrometer. In this study, unless stated otherwise, the default value of the ultrasonic frequency is $500 \mathrm{kHz}$. Note that vibration plane of the iron block was oriented at the same angle as that of the wedge incline. To match the experiment, the material for wedge and pipe in the COMSOL model were selected to be acrylic and steel, respectively. The ambient medium in the computational grid was 
chosen to be vacuum. The receiver acoustic transducer (shown with dashed lines in Figure 2) was not explicitly modeled in our computer simulations. Instead, elastic wave pressure and displacement values were obtained directly from the computational grid at observation points along the pipe.

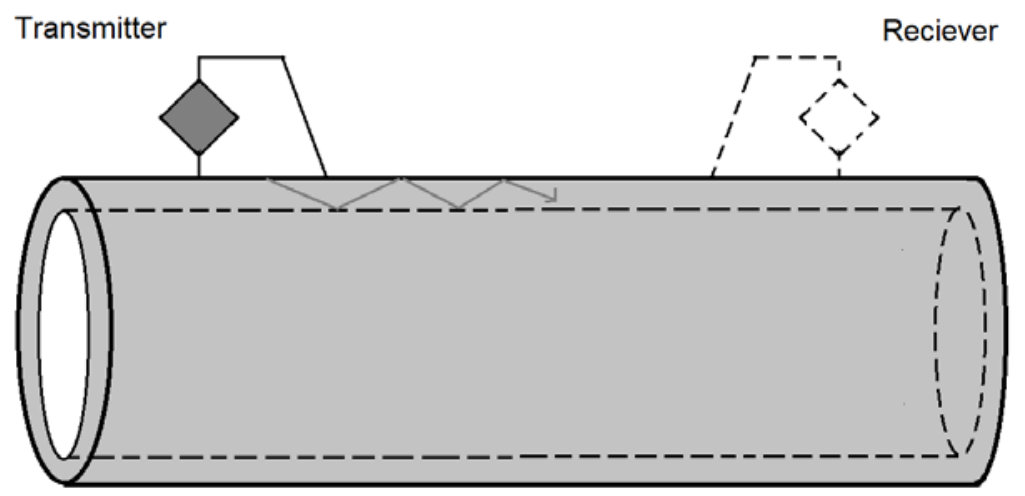

Figure 2 - Pipe-transducer model setup for computer simulation. Transducer sizes and angles are drawn not to scale. The receiver transducer (indicated with dashed lines) was not explicitly modelled in this study.

Three different approaches were pursued to model refracted shear elastic wave propagation on the piping structure. First, a two-dimensional model of radial cross section of the pipe was studied to determine radial wave propagation on the pipe. Second, a two-dimensional of refracted acoustic wave with angled beam into a steel plate was considered to investigate axial propagation of elastic waves the pipe. Finally, in the third approach, simulations for a complete three-dimensional wedge/pipe model were performed.

\subsection{Two-Dimensional Radial Cross-Section Simulations}

Elastic wave propagation on radial cross-section of a pipe was investigated for contoured wedges with different surfaces. The objective of this study was to determine how the contact area between dielectric wedge and metallic pipe affects the amplitude of elastic wave coupled into the pipe. Three different wedge contact areas were studied, as shown with color-coded combinations in Figure 3. The distance from the top of the pipe to the bottom of transducer (gray box) was held constant at $10 \mathrm{~mm}$ for all wedges. The radius of the pipe was set to $3.5 \mathrm{~cm}$ to match the experiment, The wedge with minimal contact area (black box) only had the wedge and the pipe connecting along the line at the top of the pipe. The partial contact wedge (black and red) had a contoured surface, enclosing a portion of the top arch of the pipe, with straight flanges on both sides. The total contact wedge (black, red and blue) had the entire width of the wedge contoured to match the pipe radius, and thus provided the largest contact surface area with the pipe.

Acoustic wave amplitude measured as total displacement for the three types of wedges in Figure 3 are displayed in Figure 4. The displacement obtained with each wedge in Figure 3 is plotted at different times as function of pipe circular cross-section polar angle. The top of the pipe 
corresponds to $90^{\circ}$ polar angle in these plots. Results are presented for $500 \mathrm{KHz}$ frequency. Similar patterns were observed for different frequencies. To follow time evolution of the elastic wave propagation in the structure, waves coupling into the pipe reflects off the inner surface of the pipe. For waves at normal incidence, propagation would consist of bouncing between the top and bottom surfaces of the pipe. However, most of signal wave front are incident at an angle to the vertical, Reflections at the inner pipe surface are causing the waves to propagate around the pipe in both the clockwise and counterclockwise directions. Once both of these signals reach the bottom of the pipe at $270^{\circ}$ angle in Figure 4, they begin to interfere with each other, thus potentially creating noise in the communication system.

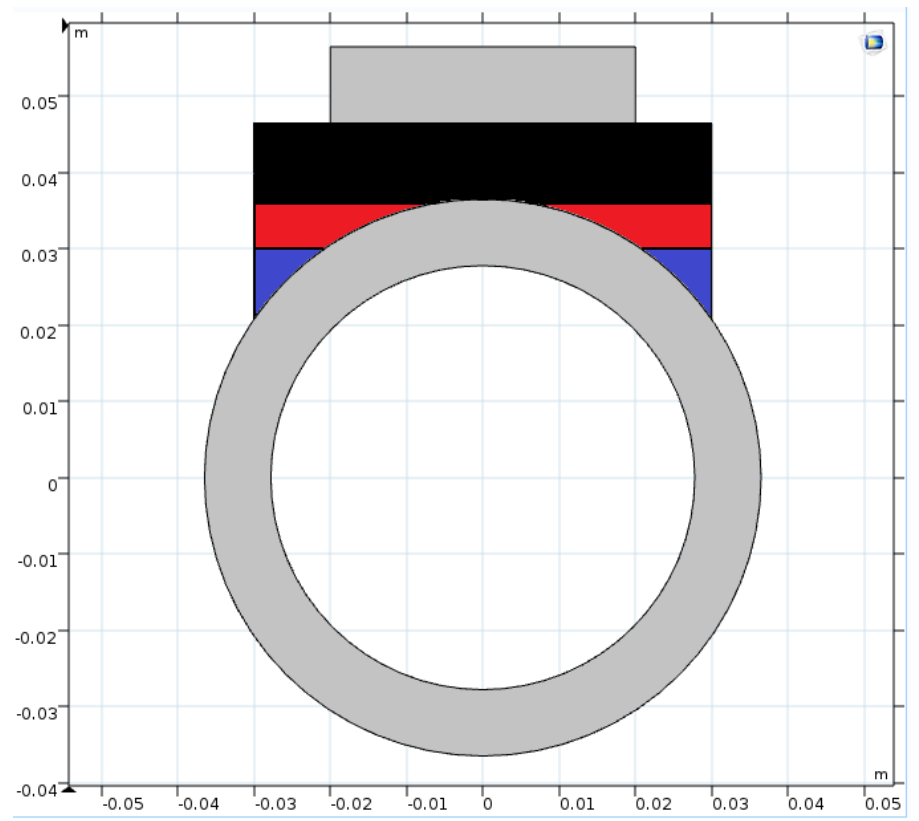

Figure 3 - Wave propagation along radial cross section of the pipe. The distance between the bottom of the transducer (gray box) and the top point of the pipe is $10 \mathrm{~mm}$. Different contact area contoured wedges are indicated with different combination of colors: Line contact (black), partial connection (black \& red), and full connection (black, red \& blue).

When different contact surface wedges were examined in Figure 4, it was concluded that as contact between the pipe and the wedge is limited, a clearer signal emerges. This result appears as a byproduct of limiting the signal's angular span of coupling into the pipe, and thus limiting the amount of reflections in the shell of the pipe. Without many reflections from the inner surface, there is less angular spread of the signal into the pipe, which could lead to a pattern of constructive and destructive interferences. However, by limiting the angular spread of the coupled beam, the total power of the acoustic signal coupled into the pipe is reduced as well.

These results suggest a trade-off between signal intensity and communication signal noise. The conclusion is that the optimal wedge is the one with partial contact (black \& red colors in Figure 3 ), where enough power is given to the signal to reach the end of the pipe, but at the same time no strong interferences are generated to create bit errors. 


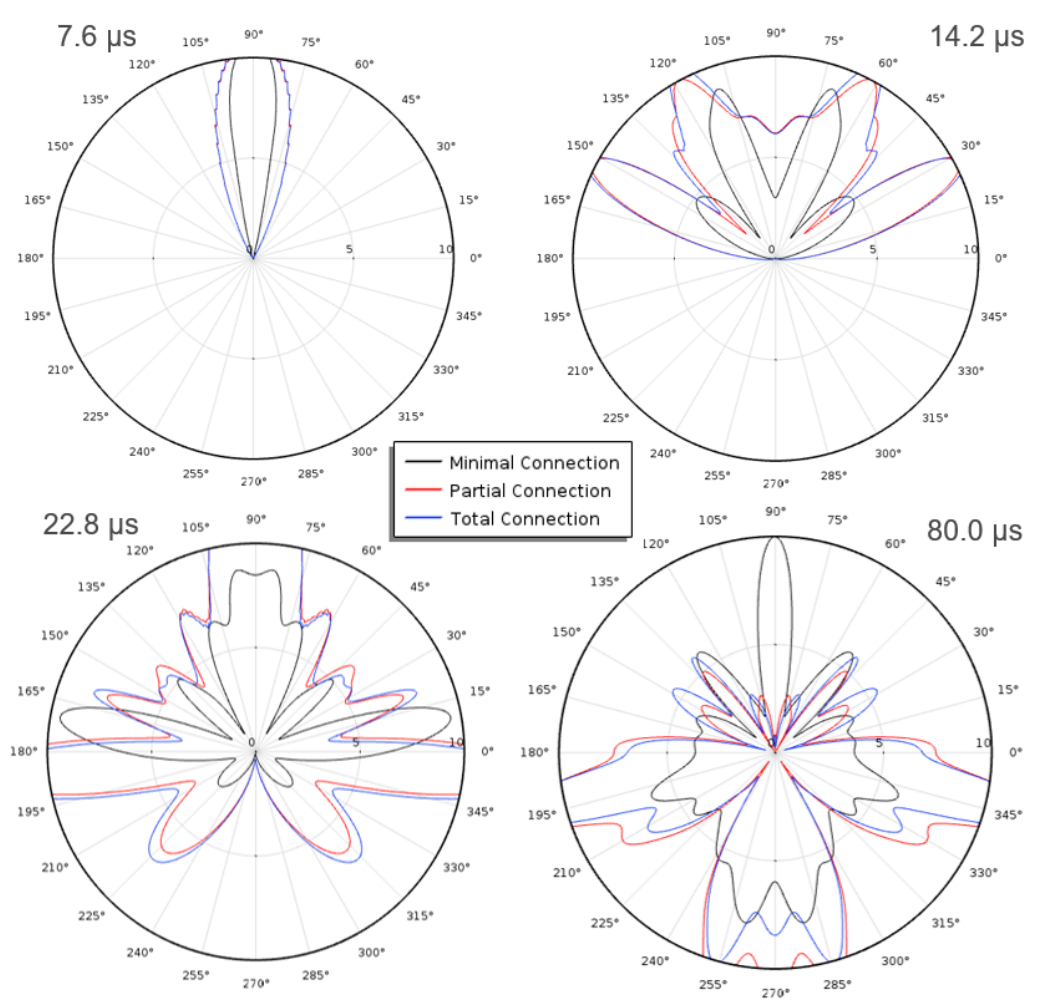

Figure 4 - Acoustic wave amplitude measured as displacement on pipe surface. The displacement obtained with each wedge in Figure 3 is plotted at different times as function of pipe circular cross-section polar angle. Results are presented for $500 \mathrm{KHz}$ frequency. Similar patterns were observed for different frequencies.

\subsection{Two-Dimensional Axial Cross-Section Simulations}

In the axial cross-section wave propagation study, wave propagation only over the top part of the pipe in contact with the transducer were considered. Thus, the cross-section of the pipe was represented by a plate with the same thickness as cylindrical shell (8.7mm to match experiment). Results of computer simulations showing acoustic wave intensity profile measured as pressure distribution after $100 \mu$ s from the start of acoustic wave transmission are shown in Figure 5. The five panels in Figure 5 show rdipaly acoustic wave intensity distribution for $500 \mathrm{KHz}, 1 \mathrm{MHz}$, $1.5 \mathrm{MHz}, 2 \mathrm{MHz}$, and $2.5 \mathrm{MHz}$ frequencies. We have observed that for some frequencies, the power of the signal coupled into the pipe was a significantly lower than that of other frequencies. Qualitatively similar phenomena has been previously observed in experiments as well. To investigate this further, multiple scattering within the wedge was studied. Within the wedge, a significant amount of the signal was reflected back from the plastic - steel boundary because of high impedance mismatch. This signal, despite the non-reflecting boundary condition on the outer walls of the wedge, reverberated continuously within the wedge. When these reflections compounded, a significant amount of scattering inside the wedge was created at higher frequencies. This is shown by comparison of acoustic pressure distribution inside the wedges at $500 \mathrm{KHz}$ and $2.5 \mathrm{MHz}$ in Figure 6. In some cases, such as $1 \mathrm{MHz}$ and $2 \mathrm{MHz}$, this caused a large 
amount of destructive interference. We believed this destructive interference attributed to some on the frequency disappearance seen in Fig. 5. We found for lower frequencies, such as $500 \mathrm{kHz}$, these reflections die out faster, as seen in Figure 6a, causing less disruption of the signal. It is unclear if the reflections within the wedge itself are a product of the simulation or are actual present in the system itself. Reflections within the wedge could be potentially suppressed by optimizing the angle with which the waves are incident at the plastic-steel boundary.

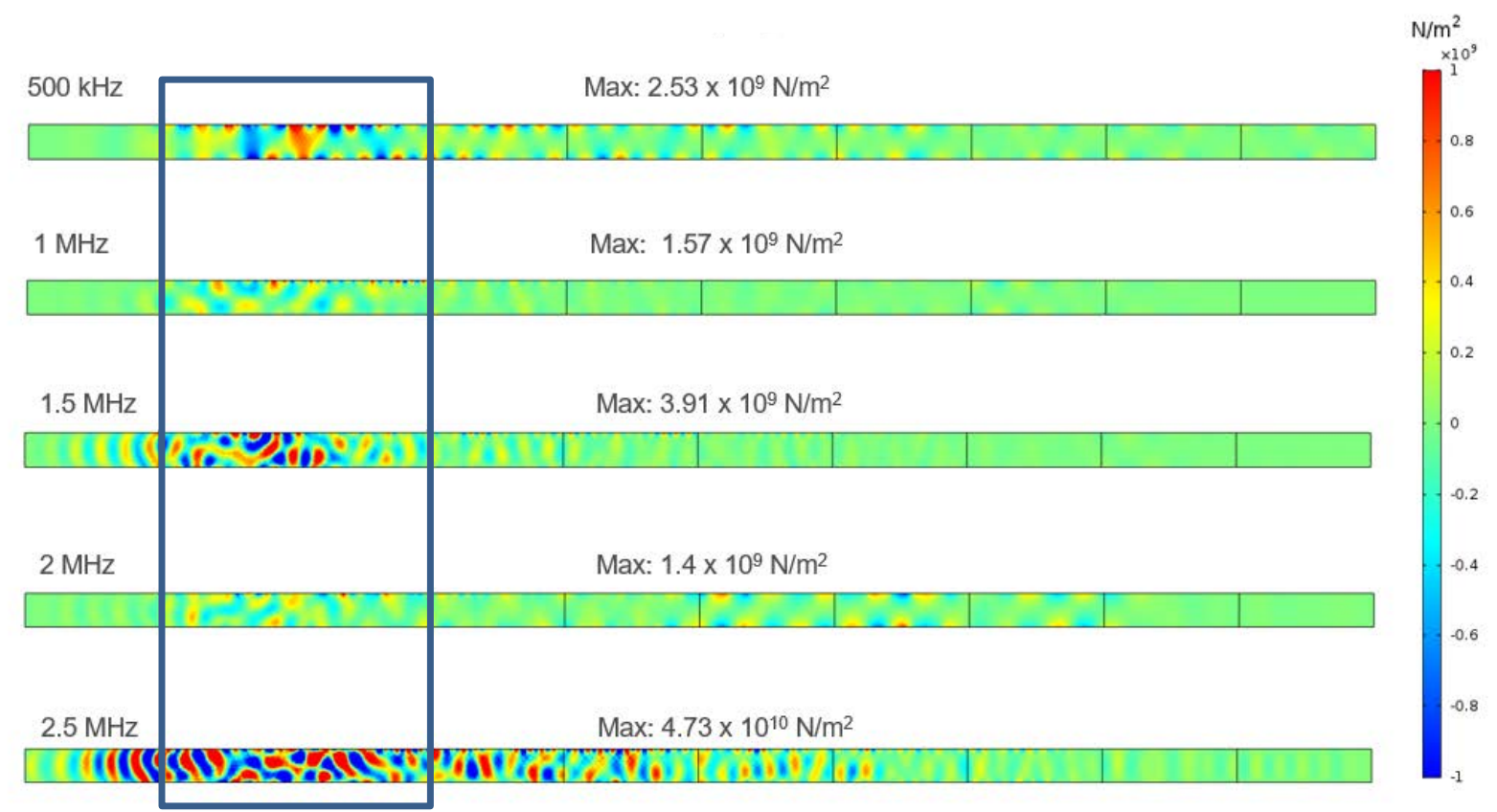

Figure 5 - Acoustic wave intensity profile measured as pressure distribution in the axial direction after $100 \mu \mathrm{s}$ of signal transmission. The wedge position is indicated with an overlaying box.

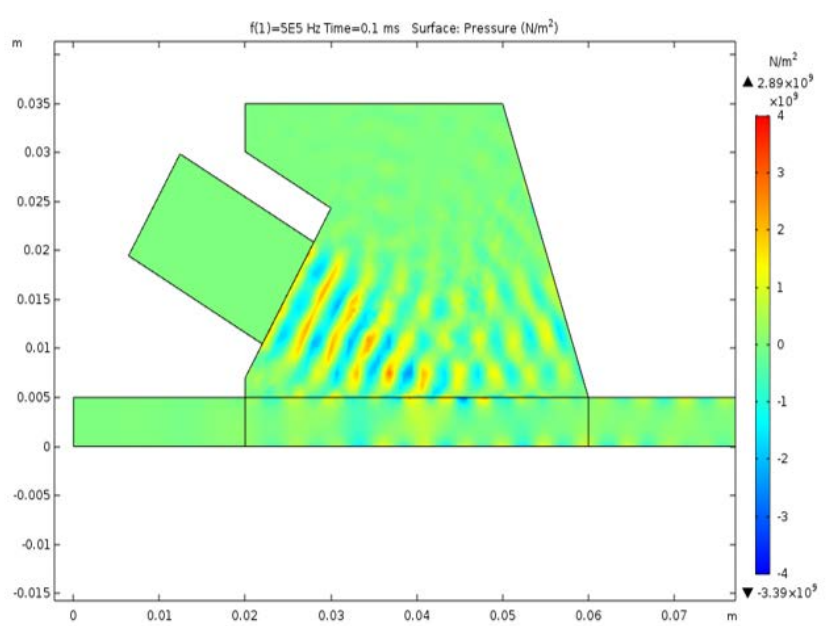

(a)

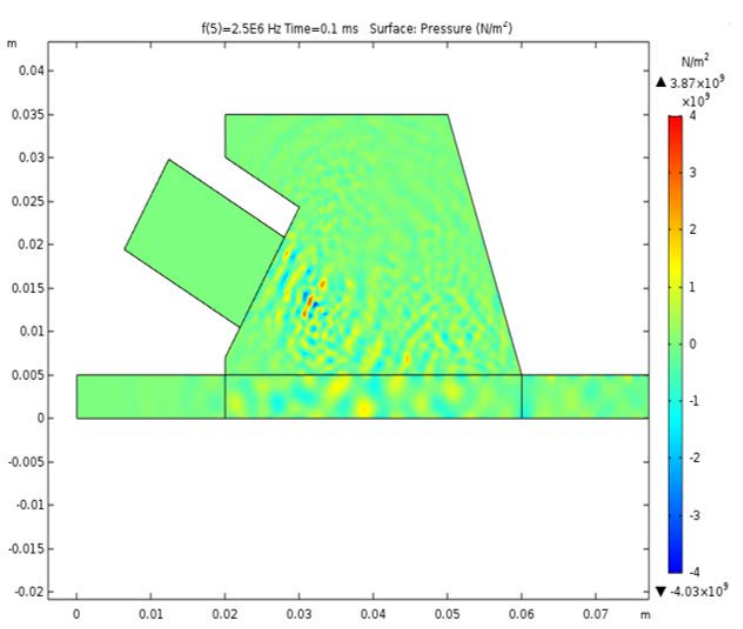

(b)

Figure 6 - Multiple reflections of acoustic wave inside the wedge for (a) $500 \mathrm{kHz}$ and (b) 2.5 MHz transducer frequency. 


\subsection{Three-Dimensional Simulations}

Three dimensional simulations were performed to model wave propagation in a section of a steel pipe of length $12.5 \mathrm{~m}$, radius of $3.5 \mathrm{~cm}$ and thickness of $8.7 \mathrm{~mm}$. The transducer was modeled as PZT represented by an iron block vibrating perpendicular to the acrylic plastic wedge surface at $500 \mathrm{kHz}$. This amplitude of displacement of the iron block was on the order of a micrometer. The wedge was assumed to be in partial contact with the pipe, as shown in Figure 3. Results of 3-D simulations which visualize distribution of elastic wave intensity are shown in Figure 7. The elastic wave intensity distributions resulting from coupling with $30^{\circ}$ and $45^{\circ}$ angled wedges are shown in Figures 7(a) and 7(b), respectively. These results indicate that 3-D propagation of elastic wave occurs in angular and axial directions, in agreement with 2-D simulations. The simulations indicate that refracted shear waves are excited in a torsional mode on the pipe, with rotations in both clockwise and counterclockwise directions. Because we were able to simulate only a small section of the pipe due to computer memory limitations, we could not visualize far field of the acoustic wave. These findings agree qualitatively with experiments which indicate that a signal could be received across the pipe when receiving PZT is positioned at $90^{\circ}$ and $180^{\circ}$ relative to the transmitting PZT. This indicates that the communication system is resilient to misalignment of transducers.

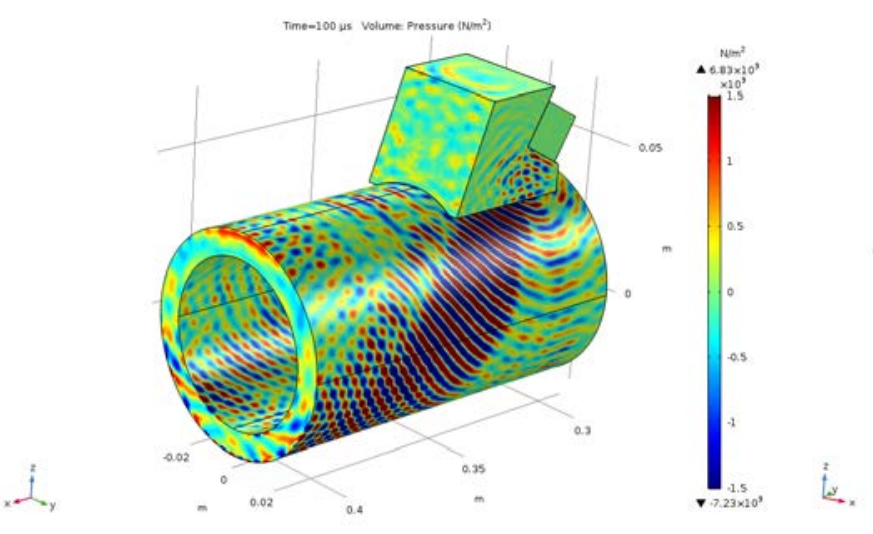

(a)

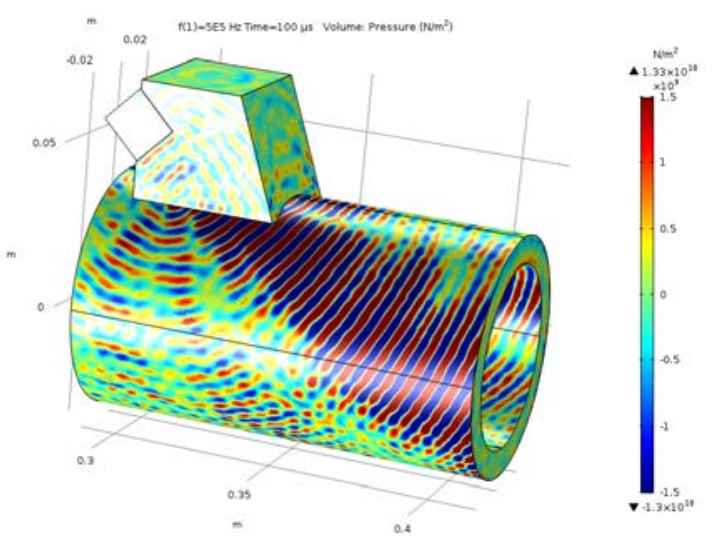

(b)

Figure 7 - Three dimensional simulation indicate torsional modes of the refracted shear wave on the pipe for excitation with (a) $30^{\circ}$ angle wedge and (b) $45^{\circ}$ angle wedge. 


\section{Resiliency of Communication System to Interferences}

Proof-of-principle studies were conducted to investigate performance of communication system under laboratory conditions designed to introduce some interferences to elastic wave propagation on a pipe, which are representative of actual deployment scenario.

\subsection{Effect of Supporting Baffle Plates on Transmission}

As outlined in previous reports on this project, the candidate pipe for acoustic communication across containment building wall is a charging line of CVCS. This pipe penetrates through a special sealed tunnel in the containment building wall. The pipe is welded to baffle plates, which are bolted to interior and exterior surfaces of the containment wall, thus sealing the tunnel (Figure 8a). In this project, we designed and fabricated stainless steel baffle plates to anchor pipe to optical bench to simulate mechanical constraints at actual NPP (Figure 8b).

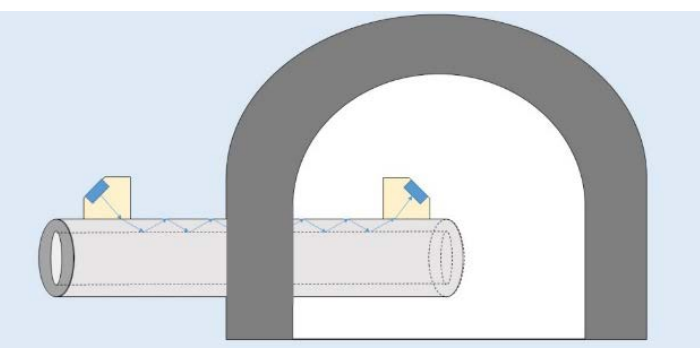

(a)

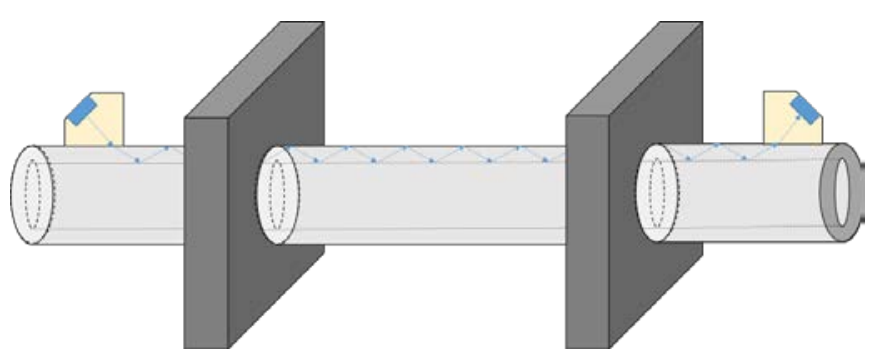

(b)

Figure 8 - (a) Envisioned deployment at NPP. (b) Laboratory bench scale setup of pipe with supporting baffle plates.

Dimensions of the stainless steel baffle plates are shown in Figure 9. Each plate was made out of two pieces, with semicircular cutout designed to fit to the pipe. Through holes for $1 / 4-20$ screws were made for assembly of the entire construction on an optical bench. When assembled, baffle plates provided support to the pipe.

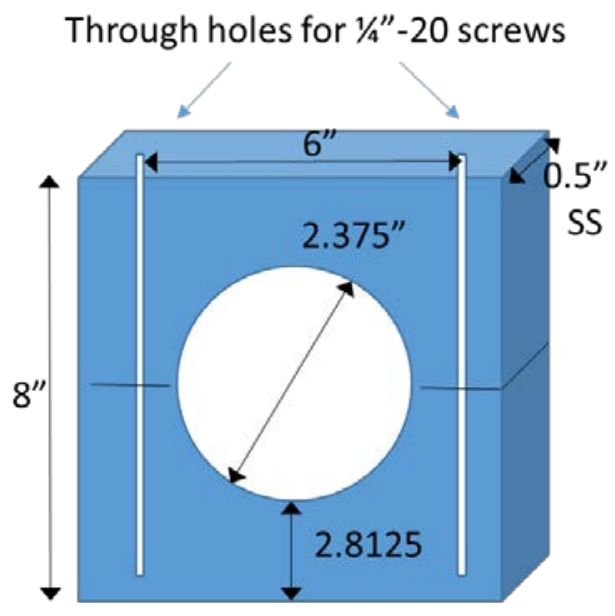

Figure 9 - Design and dimensions of each baffle plate. 
To study the effect of signal attenuation due to baffle plates, refracted shear wave signal was transmitted over the length of the pipe (1.65m), with PZT transducers positions outside the baffle plate as shown in Figure 8b. For comparison, the pipe on rested on two small cardboard boxes, and Comparison of the two signals showed slight attenuation of the acoustic signal due to baffle plates. This is to be expected, since baffle plates provide a radial support to the pipe, and little coupling of shear waves from the pipe is expected to radially attached structures.

\subsection{Effect of Coupled Low Frequency Process Noise on Signal Transmission}

Process noises due to operating machinery at a nuclear facility can potentially couple to the pipe and interfere with AC system. These noises tend be primary audible low-frequency ones, with typical bandwidth below $10 \mathrm{KHz}$.We have devised a study to investigate resilience of ultrasonic data transmission over pipe to low frequency noise. Process noise was experimentally simulated with frequency-tunable mechanical shaker placed in contact with pipe to induce low-frequency vibrations in the pipe. The photograph of the setup is shown in Figure 10.

Transmitted OOK acoustic digital signals with $200 \mu$ s bit duration at $2 \mathrm{MHz}$ shear wave carrier are shown in Figure 11. The reference acoustic signal received with mechanical shaker turned off is shown in Figure 11(a). Received acoustic signals with the pipe vibrated at $100 \mathrm{~Hz}, 1 \mathrm{KHz}, 10 \mathrm{KHz}$ are shown in Figure 11(b), 11(c) and 11(d), respectively. No significant changes in the transmitted signal caused by pipe vibration could be observed. Results of this study suggest that low-frequency process noise produces negligible interference effect on ultrasonic $2 \mathrm{MHz}$ shear wave informationcarrying signal.

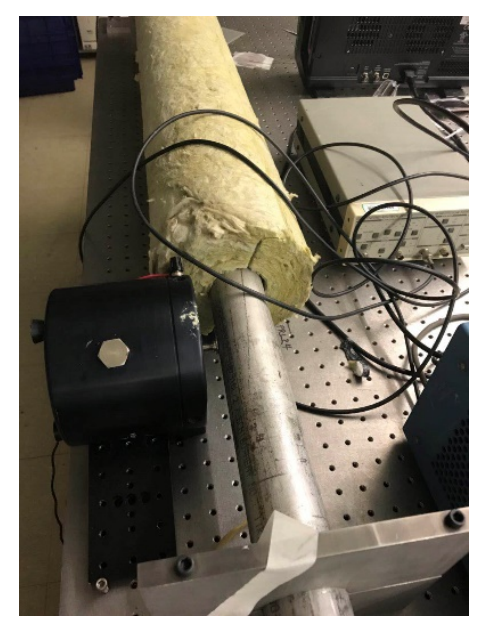

Figure 10 - Frequency-tunable mechanical shaker in contact with pipe 

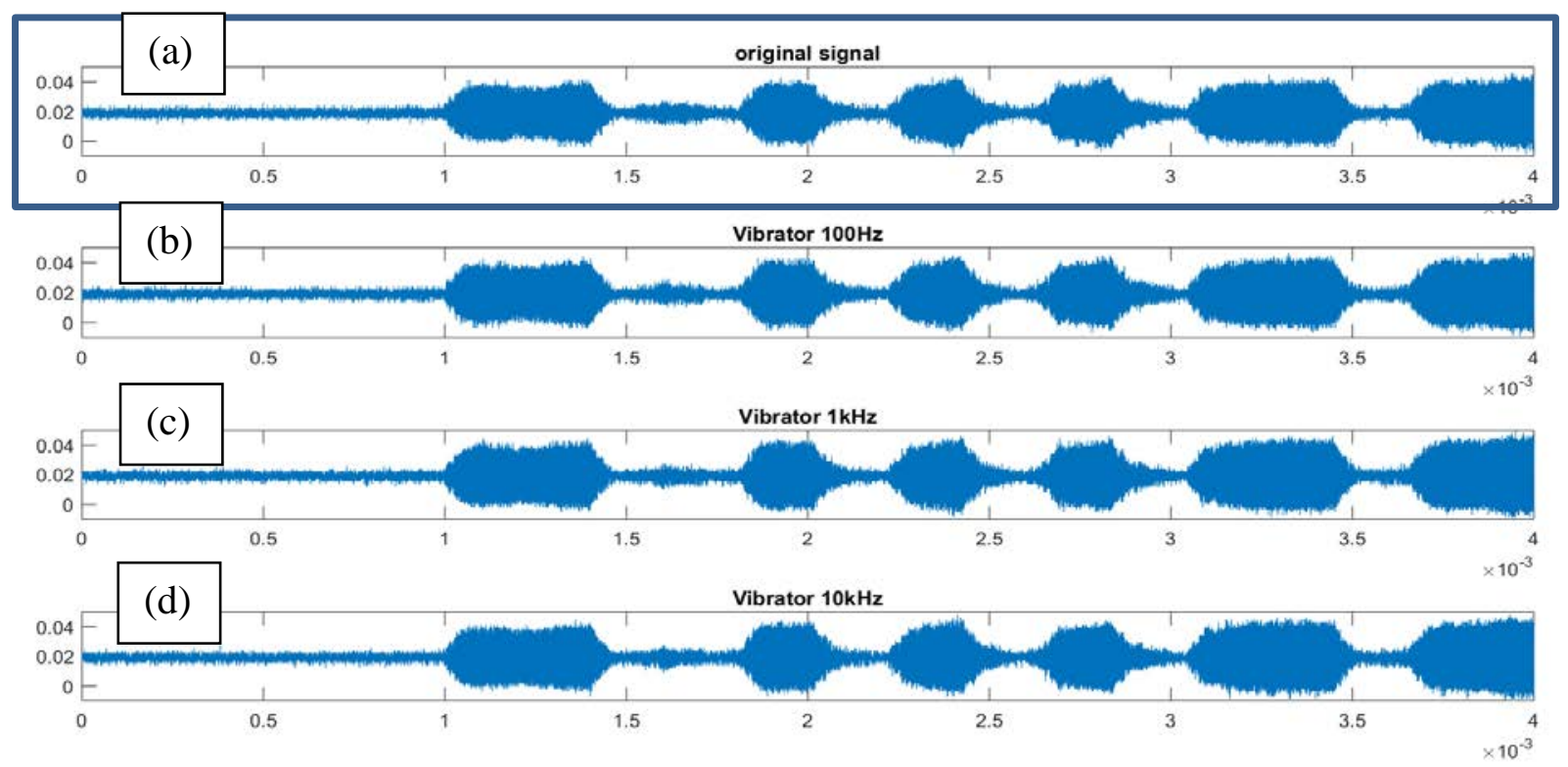

Figure 11 - Effect of mechanical vibrations on ultrasonic signal. Received acoustic signal has $2 \mathrm{MHz}$ carrier frequency and $200 \mu$ s bit pulse duration. (a) Received signal on a pipe with no vibration. (b) Received signal on pipe vibrated at $100 \mathrm{~Hz}$ (c) Received signal on pipe vibrated at $1 \mathrm{KHz}$. (d) Received signal on pipe vibrated at $10 \mathrm{KHz}$. 


\section{Conclusions}

The objective of this project is to develop and demonstrate methods for transmission of information in nuclear facilities across physical barriers as acoustic/elastic guided waves along existing in-place metal piping infrastructure. Because of limited bandwidth of ultrasonic transducers, on-off-keying OOK digital communication protocol was chosen for information transmission over an acoustic channel. Design considerations of the OOK communication protocol are intimately connected to physical characteristics of the communication channel. This report addresses acoustic channel characterization through computer modelling of elastic wave coupling and propagation in the transducer/piping structure using $\mathrm{COMSOL}^{\mathrm{TM}}$ software package. Computer simulations were performed to provide theoretical support to results obtained in laboratory experiments on signal transmission with elastic shear waves on a pipe. In particular, computer models provide qualitative analysis of coupling losses and communication signal noise due to multiple scattering at the wedge/pipe interface. Also, 3-D simulations indicate that refracted shear wave a pipe generated with an angled wedge are torsional waves, In addition, proof-of-principle experimental studies were conducted to investigate performance of communication link under the conditions closely resembling a representative scenario. Anchoring baffle plates were added to the basic setup, and low frequency process noise was simulated by mechanically shaking the pipe. Acoustic communication system was shown to be resilient against such interferences. 


\section{References}

Argonne National Laboratory (2018), "Realizing a Pipe Dream,” available at https://www.anl.gov/articles/realizing-pipe-dream

Bakhtiari, S, Chien, H.T., Heifetz A., Elmer, T.W (2018) "Nondestructive Testing Research and Development Efforts at Argonne National Laboratory: An Overview,” Materials Evaluation 76 (7), 911-920.

Heifetz A., Bakhtiari S., Huang X., Ponciroli R., and Vilim R.B. (2017) "First Annual Progress

Report on Transmission of Information by Acoustic Communication along Metal Pathways in Nuclear Facilities,” ANL/NE-17/30, September 30.

Heifetz A., Bakhtiari S., Vilim R.B. (2018) "Development of System Performance Models," Argonne National Laboratory ANL/NE-17-22.

Wang B., Saniie J., Bakhtiari S., and Heifetz A. (2017) "Architecture of an ultrasonic experimental

platform for information transmission through solids," 2017 IEEE International Ultrasonics Symposium (IUS), Washington, DC, pp. 1-4. 


\section{Argonne}

Nuclear Science and Engineering (NSE) Division

Argonne National Laboratory

9700 South Cass Avenue, Bldg. 208

Argonne, IL 60439

www.anl.gov

Argonne National Laboratory is a U.S. Department of Energy laboratory managed by UChicago Argonne, LLC 